\title{
Astaxanthin lowers plasma TAG concentrations and increases hepatic antioxidant gene expression in diet-induced obesity mice
}

\author{
Yue Yang, Tho X. Pham, Casey J. Wegner, Bohkyung Kim, Chai Siah Ku, Young-Ki Park \\ and Ji-Young Lee* \\ Department of Nutritional Sciences, University of Connecticut, Storrs, CT 06269, USA
}

(Submitted 7 May 2014 - Final revision received 16 July 2014 - Accepted 23 July 2014 - First published online 20 October 2014 )

\section{Abstract}

Non-alcoholic fatty liver disease (NAFLD) is significantly associated with hyperlipidaemia and oxidative stress. We have previously reported that astaxanthin (ASTX), a xanthophyll carotenoid, lowers plasma total cholesterol and TAG concentrations in apoE knockout mice. To investigate whether ASTX supplementation can prevent the development of NAFLD in obesity, male C57BL/6J mice ( $n 8$ per group) were fed a high-fat diet $(35 \%, \mathrm{w} / \mathrm{w})$ supplemented with $0,0.003,0.01$ or $0.03 \%$ of ASTX (w/w) for 12 weeks. The $0.03 \%$ ASTXsupplemented group, but not the other groups, exhibited a significant decrease in plasma TAG concentrations, suggesting that ASTX at a $0.03 \%$ supplementation dosage exerts a hypotriacylglycerolaemic effect. Although there was an increase in the mRNA expression of fatty acid synthase and diglyceride acyltransferase 2, the mRNA levels of acyl-CoA oxidase 1, a critical enzyme in peroxisomal fatty acid $\beta$-oxidation, exhibited an increase in the $0.03 \%$ ASTX-supplemented group. There was a decrease in plasma alanine transaminase (ALT) and aspartate transaminase (AST) concentrations in the $0.03 \%$ ASTX-supplemented group. There was a significant increase in the hepatic mRNA expression of nuclear factor erythroid 2-related factor 2 and its downstream genes, which are critical for endogenous antioxidant mechanism, in the $0.03 \%$ ASTX-supplemented group. Furthermore, there was a significant decrease in the mRNA abundance of IL-6 in the primary splenocytes isolated from the $0.03 \%$ ASTX-supplemented group upon lipopolysaccharide (LPS) stimulation when compared with that in the splenocytes isolated from the control group. In conclusion, ASTX supplementation lowered the plasma concentrations of TAG, ALT and AST, increased the hepatic expression of endogenous antioxidant genes, and rendered splenocytes less sensitive to LPS stimulation. Therefore, ASTX may prevent obesity-associated metabolic disturbances and inflammation.

\section{Key words: Astaxanthin: Non-alcoholic fatty liver disease: Hypotriacylglycerolaemia: Antioxidants: Diet-induced obesity mice}

Non-alcoholic fatty liver disease (NAFLD) is the most common cause of chronic liver diseases in Western countries ${ }^{(1)}$. It is characterised by excessive fat accumulation over 5-10\% of the total liver weight in patients with little or no history of alcohol consumption ${ }^{(2)}$. NAFLD is closely associated with increased fat deposition in the liver due to elevated lipolysis in the adipose tissue of obese subjects ${ }^{(3)}$. In approximately $10-25 \%$ of NAFLD patients, the disease progresses to nonalcoholic steatohepatitis (NASH), which is characterised by hepatocyte damage, inflammatory cell infiltration and fibrosis $^{(2,4)}$. NASH can further progress to cirrhosis and hepatocellular carcinoma ${ }^{(5)}$, increasing mortality in patients with liver diseases ${ }^{(6)}$. Currently, there is no effective therapy available for NASH patients ${ }^{(7)}$. The challenge to the development of NASH therapy is largely the complexity of the disease, which is closely related to visceral obesity, dyslipidaemia, insulin resistance and hyperglycaemia ${ }^{(3)}$.
Astaxanthin (ASTX) is a xanthophyll carotenoid that is mainly present in marine animals, such as salmon and crustaceans ${ }^{(8,9)}$. ASTX acts a potent antioxidant by functioning as a powerful reactive oxygen species quencher as well as a free radical scavenger ${ }^{(10)}$. It is known that ASTX can also scavenge peroxyl radicals and destroy peroxides, thereby protecting fatty acids and biological membranes from lipid peroxidation $^{(11,12)}$. Studies have shown that ASTX has a protective effect against oxidative stress, inflammation and metabolic disorders, such as type 2 diabetes and CVD, in animals ${ }^{(13-15)}$ and humans ${ }^{(16,17)}$. In our previous study, apoE knockout $\left(a p o E^{-/-}\right)$mice fed an atherogenic diet supplemented with ASTX exhibited decreases in the plasma concentrations of total cholesterol (TC) and $\mathrm{TAG}^{(13)}$. Furthermore, ASTX also increased the expression of nuclear factor erythroid 2-related factor 2 (NRF-2)-responsive antioxidant genes and decreased the concentrations of an oxidative

Abbreviations: ACOX-1, acyl-CoA oxidase 1; ALT, alanine transaminase; AST, aspartate transaminase; ASTX, astaxanthin; DIO, diet-induced obesity; HF, high fat; HMGR, 3-hydroxy-3-methylglutaryl-CoA reductase; LPS, lipopolysaccharide; NAFLD, non-alcoholic fatty liver disease; NASH, non-alcoholic steatohepatitis; NRF-2, nuclear factor erythroid 2-related factor 2; SREBP, sterol regulatory element-binding protein; TC, total cholesterol; TGF $\beta 1$, transforming growth factor- $\beta 1$.

*Corresponding author: Dr J.-Y. Lee, fax +1 860486 3674, email ji-young.lee@uconn.edu 
stress marker, glutathione disulphide, in the liver ${ }^{(13)}$. The study demonstrated that ASTX supplementation improves lipid metabolism as well as enhances antioxidant defence mechanisms in $a p o E^{-/-}$mice, supporting that ASTX may prevent the development of NAFLD.

In the present study, we aimed to determine the effective dietary supplementation dosage of ASTX at which it could counteract metabolic dysfunctions as well as to evaluate whether ASTX supplementation can improve metabolic and inflammatory parameters that are disturbed by a high-fat (HF) diet in diet-induced obesity (DIO) mice.

\section{Materials and methods}

\section{Animal feeding and care}

Male C57BL/6J mice aged 6 weeks were obtained from Jackson Laboratory. After 1 week of acclimation period, mice were randomly assigned to one of the following four groups: group fed a HF control diet $(35 \%, \mathrm{w} / \mathrm{w})$; group fed a HF diet supplemented with $0.003 \%$ of ASTX; group fed a HF diet supplemented with $0.01 \%$ of ASTX; group fed a HF diet supplemented with $0.03 \%$ of ASTX (by weight) ( $n 8$ per group). AstaREAL ${ }^{\circledR}$ L10, an extract from the microalgae Haematococcus pluvialis containing $10 \%$ of ASTX and approximately $90 \%$ of TAG, was kindly provided by Fuji Chemical Industry Corporation Limited. Based on body surface normalisation to a $70 \mathrm{~kg}$ individual ${ }^{(18)}$, ASTX supplementation dosages of $0.003,0.01$ and $0.03 \%$ in mice would be equivalent to approximately 20, 66 and $200 \mathrm{mg} / \mathrm{d}$ in humans. Diet composition is given in Table 1 . Mice were housed in polycarbonate cages under a $12 \mathrm{~h}$ light $-12 \mathrm{~h}$ dark cycle with free access to food and water for 12 weeks. Body weight and food intake were measured weekly. At the end of 12 weeks of feeding, mice were anaesthetised with ketamine/xylazine (120/6 mg/kg; Henry Schein Animal Health) after 8 h of fasting and then killed by cardiac puncture and cervical dislocation. Blood samples were collected in EDTA-containing vacutainers (BD Vacutainer ${ }^{\circledR}$ ) and centrifuged at $1500 \mathrm{~g}$ at $4^{\circ} \mathrm{C}$ for $15 \mathrm{~min}$

Table 1. Composition of the high-fat (HF) control diet and the HF diets containing astaxanthin (ASTX)*

\begin{tabular}{lrrrr}
\hline & & \multicolumn{3}{c}{ ASTX } \\
\cline { 3 - 5 } Composition $(\mathrm{g} / \mathrm{kg})$ & Control† & $0.003 \%$ & $0.01 \%$ & $0.03 \%$ \\
\hline Dextrinised maize starch & 162.6 & 162.6 & 162.6 & 162.6 \\
Sucrose & 89.5 & 89.5 & 89.5 & 89.5 \\
Casein & 260.1 & 260.1 & 260.1 & 260.1 \\
L-Cystine & 3.9 & 3.9 & 3.9 & 3.9 \\
Coconut oil & 318.7 & 318.7 & 318.7 & 318.7 \\
Soyabean oil & 32.5 & 32.2 & 31.5 & 29.5 \\
Insoluble fibre & 65.0 & 65.0 & 65.0 & 65.0 \\
AIN-93 mineral mix & 52.0 & 52.0 & 52.0 & 52.0 \\
AIN-93 vitamin mix & 13.0 & 13.0 & 13.0 & 13.0 \\
Choline bitartrate & 2.6 & 2.6 & 2.6 & 2.6 \\
tert-Butylhydroquinone & 0.01 & 0.01 & 0.01 & 0.01 \\
AstaREAL ${ }^{\circledR}$ L10 & 0 & 0.3 & 1.0 & 3.0 \\
Total & 1000.0 & 1000.0 & 1000.0 & 1000.0 \\
\hline
\end{tabular}

${ }^{*}$ ASTX supplementation dosages by weight.

†The HF control diet contained $35 \%$ fat by weight. to remove erythrocytes. Liver and epididymal and retroperitoneal adipose tissue samples were snap-frozen in liquid $\mathrm{N}_{2}$ and stored at $-80^{\circ} \mathrm{C}$ until use. A small portion of the tissue samples was fixed in 10\% formalin for histological analysis. All procedures were approved by the Institutional Animal Care and Use Committee of the University of Connecticut.

\section{Plasma chemistry and hepatic lipid quantification}

Lipid from liver samples was extracted using the Folch method as described previously ${ }^{(19)}$. Plasma and hepatic lipids were quantified using enzymatic assays for TC, TAG, and HDLcholesterol, and plasma glucose was quantified using the Liquid Glucose (Oxidase) Reagent Set (Pointe Scientific, Inc.) ${ }^{(20)}$. Plasma insulin was quantified using ELISA (Alpco) according to the manufacturer's instructions. Plasma alanine transaminase (ALT) and aspartate transaminase (AST) were quantified using the Cholestech LDX System (Cholestech Corporation).

\section{Gene expression analysis}

Total RNA was isolated from liver and adipose tissue samples as described previously using TRIzol Reagent (Invitrogen) ${ }^{(21)}$. Quantitative real-time PCR analysis was carried out as described previously using the SYBR Green procedure and CFX96 Touch $^{\text {тм }}$ Real-Time PCR Detection System (Bio$\mathrm{Rad})^{(22)}$. Primer sequences were designed according to the GenBank database using the Beacon Designer software (PREMIER Biosoft), and primer sequences are available upon request. Ribosomal protein, large, P0 was used as an internal control for data normalisation.

\section{Primary splenocyte isolation and treatment}

Primary splenocytes were isolated from freshly isolated spleens as described previously ${ }^{(23)}$. The splenocytes were resuspended in RPMI-1640 medium containing 10\% fetal bovine serum, $100 \mathrm{U} / \mathrm{ml}$ penicillin and $100 \mu \mathrm{g} / \mathrm{ml}$ streptomycin and were then stimulated with $100 \mathrm{ng} / \mathrm{ml}$ lipopolysaccharide (LPS) for $18 \mathrm{~h}$ for gene expression analysis.

\section{Histological evaluation of liver samples}

Formalin-fixed liver samples were sliced into $5 \mu \mathrm{m}$-thick sections and stained with haematoxylin and eosin by the Connecticut Veterinary Medical Diagnostic Laboratory. The stained tissue sections were examined using a Zeiss Axio Observer.A1 microscope (Carl Zeiss Microscopy), and images were obtained at $20 \times$ magnification with an AxioCam MRc camera (Carl Zeiss Microscopy).

\section{Statistical analysis}

One-way ANOVA or Student's unpaired $t$ test was carried out using GraphPad Prism 6 (GraphPad Software) to identify statistically significant differences between the groups with a two-tailed level of significance at $P<0 \cdot 05$. Normality and equity of variance were tested using D'Agostino-Pearson 
Table 2. Body and tissue weights and plasma insulin and glucose concentrations of C57BL/6J mice fed a high-fat (HF) control diet or a HF diet supplemented with $0.03 \%(\mathrm{w} / \mathrm{w})$ of astaxanthin (ASTX) for 12 weeks (Mean values with their standard errors; $n 5-8$ )

\begin{tabular}{|c|c|c|c|c|}
\hline & \multicolumn{2}{|c|}{ Control } & \multicolumn{2}{|c|}{$0.03 \%$ ASTX } \\
\hline & Mean & SEM & Mean & SEM \\
\hline Body weight (g) & $39 \cdot 3$ & $2 \cdot 43$ & $41 \cdot 0$ & 1.67 \\
\hline Total weight gain $(\mathrm{g})$ & $15 \cdot 9$ & $2 \cdot 00$ & 18.4 & 1.44 \\
\hline $\begin{array}{l}\text { Epididymal adipose } \\
\text { tissue weight }(\mathrm{g})\end{array}$ & 1.80 & 0.23 & $2 \cdot 62^{*}$ & 0.18 \\
\hline $\begin{array}{l}\text { Retroperitoneal adipose } \\
\text { tissue weight }(\mathrm{g})\end{array}$ & 0.50 & 0.06 & 0.67 & 0.06 \\
\hline $\begin{array}{l}\text { Plasma fasting glucose } \\
\text { concentration }(\mathrm{mmol} / \mathrm{l})\end{array}$ & $17 \cdot 9$ & $2 \cdot 67$ & $19 \cdot 8$ & $1 \cdot 13$ \\
\hline $\begin{array}{l}\text { Plasma fasting insulin } \\
\text { concentration }(\mathrm{pmol} / \mathrm{l})\end{array}$ & 172 & $6 \cdot 49$ & 177 & $10 \cdot 6$ \\
\hline
\end{tabular}

${ }^{\star}$ Mean value was significantly different from that of the control group $(P<0.05)$.

omnibus normality test and Bartlett's test, respectively. Data are expressed as means with their standard errors.

\section{Results}

\section{Body and tissue weights and plasma chemistry}

There were no significant differences in food intake (data not shown), body weight, body weight gain and retroperitoneal adipose tissue weight between the control and 0.03\% ASTX-supplemented groups (Table 2). However, the 0.03\% ASTX-supplemented group exhibited a significantly higher epididymal adipose tissue weight when compared with the control group. There were no significant differences in plasma glucose and insulin concentrations between the control and 0.03\% ASTX-supplemented groups.

\section{Hypotriacylglycerolaemic effect of $0.03 \%$ astaxanthin supplementation in diet-induced obesity mice}

We have previously reported that $0.03 \%$ ASTX supplementation lowers plasma TC and TAG concentrations in

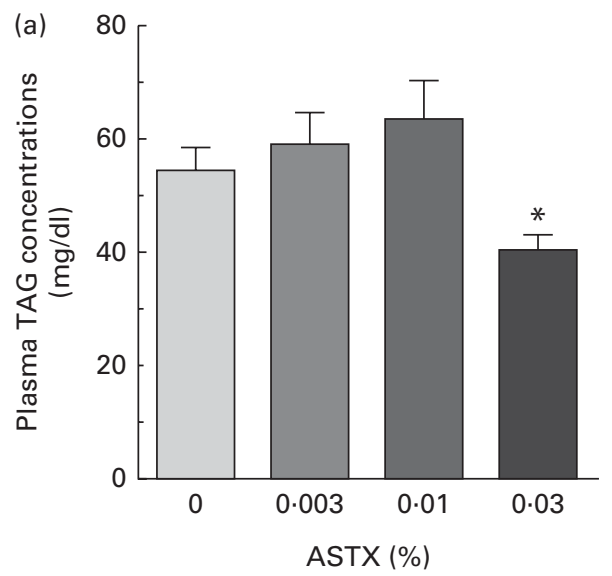

$a p o E^{-1-}$ mice fed an atherogenic diet ${ }^{(13)}$. To determine the effective supplementation dosage of ASTX at which it could lower blood lipid concentrations in obesity, mice were fed a HF diet supplemented with $0.003,0.01$ or $0.03 \%$ of ASTX (w/w). The $0.03 \%$ ASTX-supplemented group, but not the 0.003 and $0.01 \%$ ASTX-supplemented groups, exhibited significantly lower plasma TAG concentrations when compared with the control group (Fig. 1(a)). There were no significant differences in plasma TC and HDL-cholesterol concentrations between the control and 0.03\% ASTX-supplemented groups (Fig. 1(b)). These results suggest that ASTX supplementation at a dosage of $0.03 \%$ is required to elicit a TAG-lowering effect in DIO mice. Therefore, only the $0.03 \%$ ASTXsupplemented group was used for subsequent analyses to compare with the control group.

\section{Effect of $0.03 \%$ astaxanthin supplementation on hepatic lipid concentrations and damage}

There were no significant differences in liver weight and hepatic TAG and TC concentrations between the control and 0.03\% ASTX-supplemented groups (Fig. 2(a)). Consistently, histological analysis of haematoxylin and eosin-stained liver sections showed no noticeable difference in lipid accumulation between the two groups (Fig. 2(b)). However, a trend towards lower plasma ALT concentrations $(P=0.071)$ and significantly lower plasma AST concentrations $(P=0.015)$ was observed in the $0.03 \%$ ASTX-supplemented group when compared with the control group (Fig. 2(c)).

\section{Effect of astaxanthin supplementation on the hepatic expression of lipogenic, cholesterologenic, inflammatory and fibrogenic genes}

To gain mechanistic insights into the TAG-lowering effect of ASTX, the expression of genes involved in lipogenesis and fatty acid $\beta$-oxidation was measured in the liver. There were no differences in the mRNA abundance of sterol regulatory element-binding protein $(S R E B P)-1 c$, a key transcription

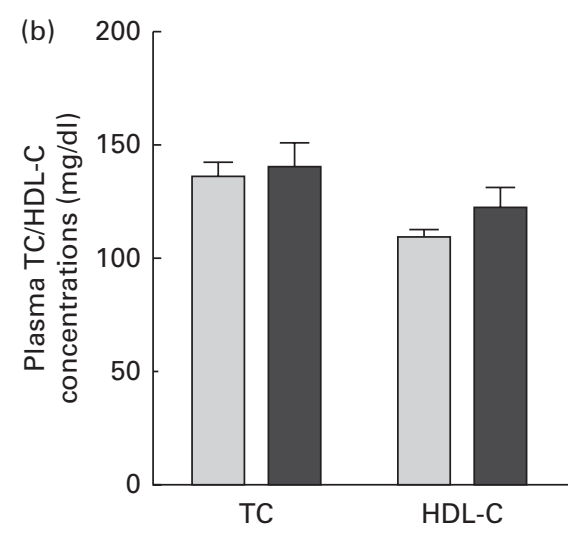

Fig. 1. Plasma lipid profiles. Male C57BL/6J mice were fed a high-fat (HF) control diet ( $\square$ ) or a HF diet supplemented with $0.003,0.01$ or $0.03 \%$ of astaxanthin ( $\square$ ) by weight for 12 weeks. (a) Plasma concentrations of TAG and (b) total cholesterol (TC) and HDL-cholesterol (HDL-C). Values are means, with their standard errors represented by vertical bars $(n 5-8)$. * Mean value was significantly different from that of the control group $(P<0.05)$. To convert TAG in mg/dl to mmol/l, multiply by 0.01129 . To convert cholesterol in $\mathrm{mg} / \mathrm{dl}$ to $\mathrm{mmol} / \mathrm{l}$, multiply by 0.02586 . 
(a)

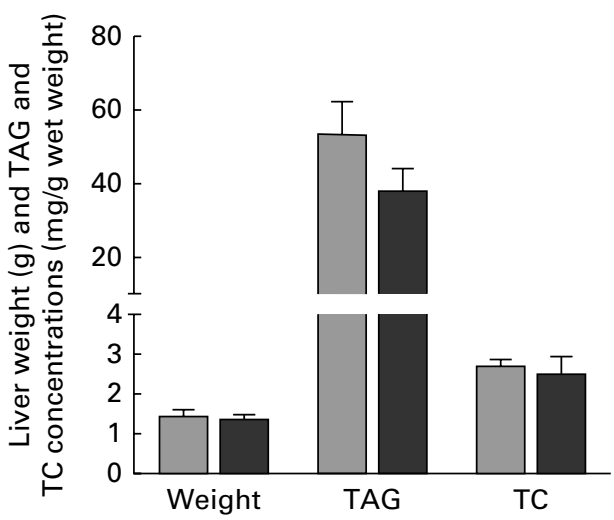

(c)

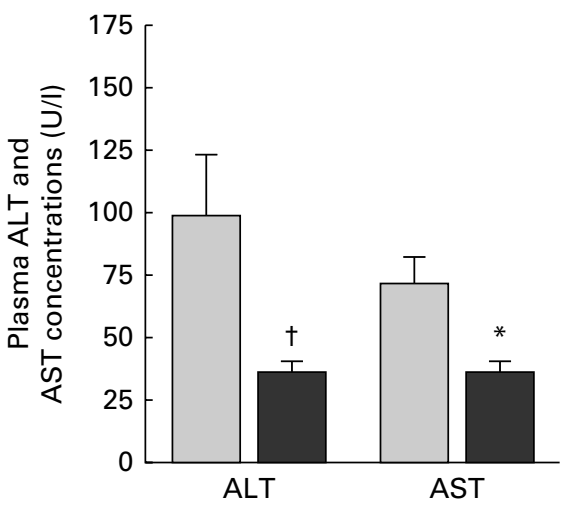

(b)
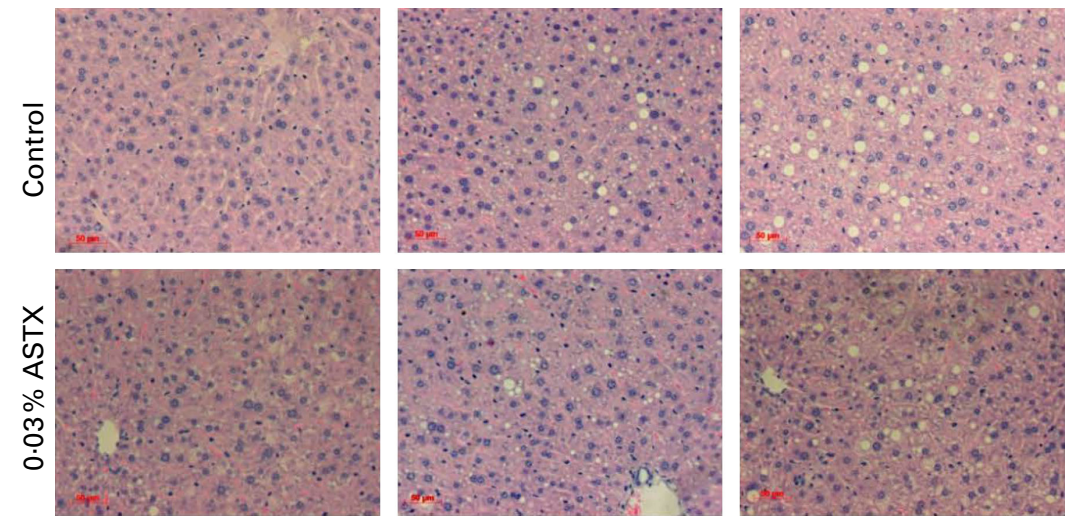

Fig. 2. (a) Liver weight and TAG and total cholesterol (TC) concentrations. (b) Representative liver sections stained with haematoxylin and eosin at 20x magnification. (c) Plasma alanine transaminase (ALT) and aspartate transaminase (AST) concentrations. Male C57BL/6J mice were fed a high-fat (HF) control diet ( $\square$ ) or a HF diet supplemented with $0.03 \%$ of astaxanthin (ASTX, $\square$ ) by weight for 12 weeks. Values are means, with their standard errors represented by vertical bars $(n 5-8)$. * Mean value was significantly different from that of the control group $(P<0.05)$. † Mean value was tended to be decreased from that of control group $(P<0.05)$.

factor in lipogenesis, and stearoyl-CoA desaturase 1 between the control and $0.03 \%$ ASTX-supplemented groups, while there was a significant increase in the mRNA abundance of fatty acid synthase and diglyceride acyltransferase 2 in ASTX-supplemented mice than in control mice (Table 3). In contrast to lipogenic genes, there was a significant increase in the mRNA expression of acyl-CoA oxidase 1 (ACOX-1), the rate-limiting enzyme in the peroxisomal fatty acid $\beta$-oxidation, in the $0.03 \%$ ASTX-supplemented group, while there was no change in the mRNA expression of carnitine palmitoyltransferase- $1 \alpha$, the rate-limiting enzyme in mitochondrial fatty acid $\beta$-oxidation. Although there were no significant differences in plasma TC and HDL-cholesterol concentrations between the groups, marginal but significant increases in the hepatic expression of 3-hydroxy-3-methylglutaryl-CoA reductase $(H M G R)$ and LDL receptor were observed in the $0.03 \%$ ASTX-supplemented group with no difference being observed in that of SREBP-2. There were no significant differences in the expression of genes involved in HDL metabolism, including scavenger receptor class B, type 1 and apoA-I, between the control and $0.03 \%$ ASTX-supplemented groups.
Inflammation with increased macrophage infiltration and elevated fibrogenesis in the liver is a major marker of $\mathrm{NASH}^{(24)}$. The mRNA expression of $\alpha$-smooth muscle actin and procollagen type I, $\alpha 1$, which are markers of fibrosis, was not altered in the $0.03 \%$ ASTX-supplemented group, while that of transforming growth factor- $\beta 1$ (TGF $\beta 1$ ), a potent profibrogenic cytokine, was significantly increased (Table 3). There was no change in the mRNA expression of F4/80, a macrophage marker, in ASTX-supplemented mice.

Effect of astaxanthin supplementation on the expression of nuclear factor erythroid 2-related factor 2 and its target genes in the liver

As ASTX is a well-known antioxidant, we examined whether ASTX supplementation can alter a NRF2-mediated endogenous antioxidant defence system. There was a significant increase in the mRNA expression of $N R F-2$ as well as its target genes, including superoxide dismutase 1, glutamatecysteine ligase regulatory subunit and glutathione peroxidase 1, in the liver of the $0.03 \%$ ASTX-supplemented group than in the control group (Fig. 3). 
Table 3. Hepatic and adipose tissue gene expression of C57BL/6J mice fed a high-fat (HF) control diet or a HF supplemented with $0.03 \%(\mathrm{w} / \mathrm{w})$ of astaxanthin (ASTX) for 12 weeks

(Mean values with their standard errors; $n 5-8$ )

\begin{tabular}{|c|c|c|c|c|c|c|}
\hline \multirow[b]{2}{*}{ Function } & \multirow[b]{2}{*}{ Gene } & \multicolumn{2}{|c|}{ Control } & \multicolumn{2}{|c|}{$0.03 \%$ ASTX } & \multirow[b]{2}{*}{$P$} \\
\hline & & Mean & SEM & Mean & SEM & \\
\hline \multicolumn{7}{|l|}{ Liver } \\
\hline \multirow[t]{4}{*}{ Lipogenesis } & $S R E B P-1 C$ & 1.07 & 0.15 & 1.58 & 0.25 & 0.092 \\
\hline & FAS & 1.06 & 0.14 & 1.59 & 0.19 & 0.040 \\
\hline & $S C D-1$ & 1.31 & 0.28 & 1.53 & 0.43 & 0.662 \\
\hline & $D G A T-2$ & $1 \cdot 16$ & 0.19 & $2 \cdot 28$ & 0.08 & 0.003 \\
\hline \multirow{2}{*}{ Fatty acid oxidation } & $A C O X-1$ & 1.02 & 0.07 & 1.42 & 0.09 & 0.009 \\
\hline & $C P T-1 \alpha$ & 1.09 & 0.17 & 1.23 & 0.12 & 0.583 \\
\hline \multirow[t]{3}{*}{ Cholesterol metabolism } & $S R E B P-2$ & 1.11 & 0.19 & 0.92 & 0.18 & 0.490 \\
\hline & HMGR & 1.06 & 0.14 & 1.59 & 0.19 & 0.040 \\
\hline & $L D L R$ & 1.13 & 0.22 & $2 \cdot 04$ & 0.40 & 0.048 \\
\hline \multirow{2}{*}{ HDL metabolism } & $S R-B 1$ & $1 \cdot 10$ & 0.17 & 1.45 & 0.36 & 0.346 \\
\hline & ApoA-I & 1.01 & 0.11 & 1.28 & 0.26 & 0.468 \\
\hline \multirow[t]{4}{*}{ Inflammation and fibrosis } & $\alpha-S M A$ & 1.08 & 0.18 & 0.63 & 0.18 & 0.110 \\
\hline & Col1A1 & 1.14 & 0.20 & 0.83 & 0.09 & 0.332 \\
\hline & $T G F \beta 1$ & 1.09 & 0.17 & 4.50 & 1.03 & 0.002 \\
\hline & $F 4 / 80$ & 1.04 & 0.23 & 1.25 & 0.26 & 0.550 \\
\hline \multicolumn{7}{|l|}{ Epididymal adipose tissue } \\
\hline \multirow[t]{2}{*}{ Lipogenesis } & $S R E B P-1 C$ & $1 \cdot 10$ & 0.20 & 1.42 & 0.26 & 0.338 \\
\hline & FAS & 1.21 & 0.23 & 1.34 & 0.24 & 0.716 \\
\hline \multirow[t]{2}{*}{ Inflammation } & $T N F \alpha$ & 1.74 & 0.56 & 1.52 & 0.44 & 0.789 \\
\hline & $F 4 / 80$ & $2 \cdot 61$ & 1.08 & 0.70 & 0.13 & 0.250 \\
\hline
\end{tabular}

$S R E B P$, sterol regulatory element-binding protein; $F A S$, fatty acid synthase; $S C D-1$, stearoyl-CoA desaturase 1 ; $D G A T-2$, diglyceride acyltransferase $2 ; A C O X-1$, acyl-CoA oxidase $1 ; C P T-1 \alpha$, carnitine palmitoyltransferase-1 $\alpha$; HMGR, 3-hydroxy-3-methylglutaryl-CoA reductase; $L D L R$, LDL receptor; $S R-B 1$, scavenger receptor class $\mathrm{B}$, type 1; $\alpha$-SMA, $\alpha$-smooth muscle actin; Col1A1, procollagen type I, $\alpha 1$; TGF $\beta 1$, transforming growth factor- $\beta 1$.

\section{Effect of $0.03 \%$ astaxanthin supplementation on} the inflammatory responses of splenocytes

It has been suggested that the spleen functions as a reservoir of monocytes ${ }^{(25)}$. To determine whether ASTX supplementation alters the inflammatory response of splenocytes, the primary cells isolated from control and $0.03 \%$ ASTX-supplemented mice were stimulated with LPS. There was a significant decrease in the mRNA abundance of $I L-6$ in splenocytes isolated from $0.03 \%$ ASTX-supplemented mice $(P=0.038)$ when compared with that in splenocytes isolated from control mice, while there were no differences in the mRNA abundance of $T N F \alpha$ between the groups (Fig. 4)

\section{Discussion}

Lipotoxicity, oxidative stress and inflammation are commonly identified as key players in the pathogenesis of $\mathrm{NASH}^{(26-28)}$. Obesity is also a major risk factor for NAFLD and NASH development $^{(29)}$. In obesity, fatty acid flux to the liver is increased

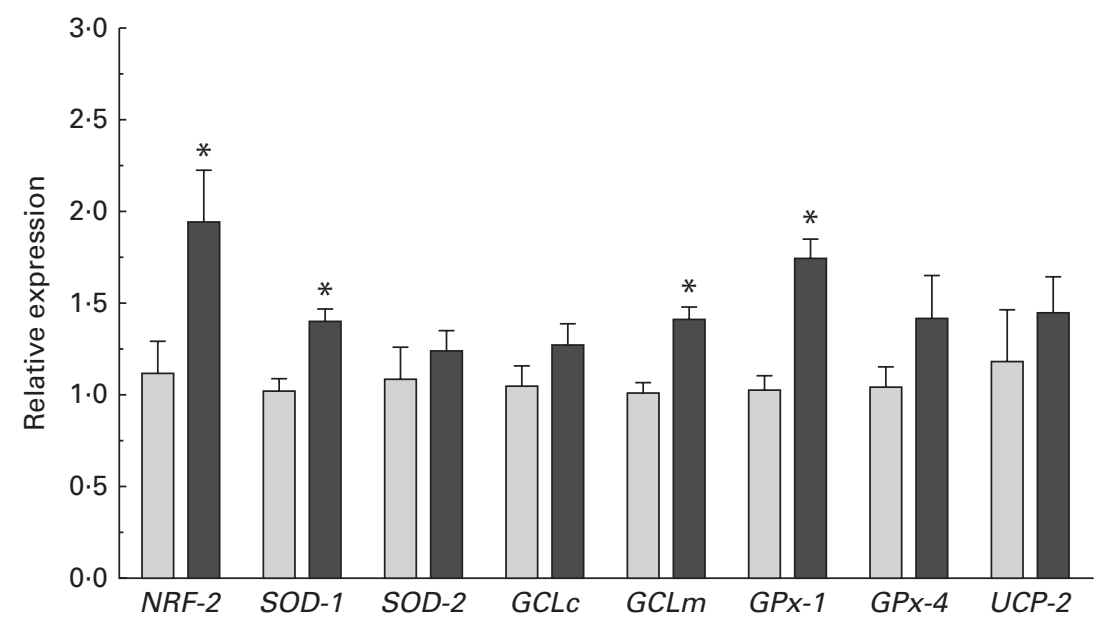

Fig. 3. Hepatic mRNA expression of antioxidant genes. Male C57BL/6J mice were fed a high-fat (HF) control diet ( $\square$ ) or a $\mathrm{HF}$ diet supplemented with $0.03 \%$ of astaxanthin $(\square)$ by weight for 12 weeks. Values are means, with their standard errors represented by vertical bars ( $n$ 8). ${ }^{*}$ Mean value was significantly different from that of the control group $(P<0.05)$. NRF-2, nuclear factor erythroid 2-related factor 2; $S O D$, superoxide dismutase; GCLc, glutamate-cysteine ligase, catalytic subunit; GCLm, glutamate-cysteine ligase regulatory subunit; GPX, glutathione peroxidase; UCP-2, uncoupling protein 2. 


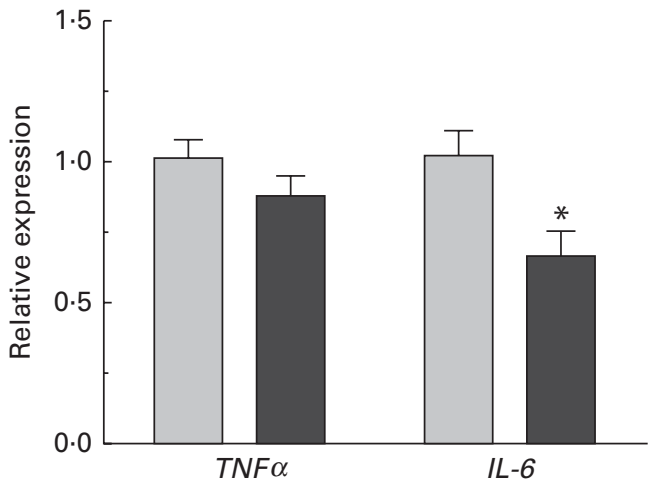

Fig. 4. Inflammatory response of splenocytes. Splenocytes were isolated from male C57BL/6J mice fed a high-fat (HF) control diet ( $\square$ ) or a HF diet supplemented with $0.03 \%$ of astaxanthin $(\square)$ by weight for 12 weeks and stimulated with lipopolysaccharide $(100 \mathrm{ng} / \mathrm{ml})$ for $18 \mathrm{~h}$ for quantitative realtime PCR analysis. Values are means, with their standard errors represented by vertical bars $(n 8)$. ${ }^{*}$ Mean value was significantly different from that of the control group $(P<0.05)$.

due to elevated lipolysis in hypertrophied adipocytes, leading to the accumulation of excessive TAG, oxidative stress, inflammation and insulin resistance in the liver. Insulin resistance promotes de novo lipogenesis and gluconeogenesis, further contributing to the development of dyslipidaemia, hyperglycaemia and other metabolic disorders, such as type 2 diabetes and $\mathrm{CVD}^{(30)}$. The goal of the present study was to determine the effective supplementation dosage of ASTX at which it could counteract metabolic dysfunctions, oxidative stress and inflammation induced by a HF diet in DIO mice. We found that ASTX at a dosage of $0.03 \%$ exerted a TAG-lowering effect, increased hepatic expression of endogenous antioxidant genes with a concomitant decrease in steatosis, and attenuated the inflammatory response of splenocytes to LPS in DIO mice. In addition, it should be noted that ASTX lowered plasma ALT and AST concentrations in DIO mice. ALT is predominately found in the liver, whereas AST is present in the liver, cardiac and skeletal muscle, kidneys, brain and erythrocytes ${ }^{(31)}$. Therefore, the reduction in plasma ALT and AST concentrations by ASTX suggests that it may prevent not only liver damage, but also potential HF-induced tissue damage in other organs.

Hypertriacylglycerolaemia, a common lipid abnormality in obese patients, is a major contributor of the development of insulin resistance ${ }^{(32)}$, type 2 diabetes ${ }^{(33)}, \mathrm{CVD}^{(34)}$ and NAFLD $^{(35)}$. Hypertriacylglycerolaemia in obesity primarily occurs due to increased hepatic de novo lipogenesis consequent to elevated NEFA flux into the liver from dysfunctional adipocytes $^{(30)}$. Consistent with our previous observation that $0.03 \%$ ASTX supplementation significantly lowers plasma TAG concentrations in $a p o E^{-/-}$mice ${ }^{(13)}$, ASTX at the same supplementation dosage also decreased plasma TAG concentrations in DIO mice in the present study. A hypotriacylglycerolaemic effect of ASTX has also been reported in humans ${ }^{(17)}$. In both $a p o E^{-1-}$ and DIO mice, we observed a significant increase in the hepatic mRNA expression of $A C O X-1$, while the expression of lipogenic genes was not altered or increased by ASTX supplementation. Hepatic TAG concentrations in $0.03 \%$ ASTX-supplemented mice were lower by approximately $30 \%$ when compared with those in control mice, although they did not reach statistical significance. Therefore, it can be presumed that elevated peroxisomal fatty acid oxidation due to increased $A C O X-1$ expression induced by ASTX may be responsible for the lipid-lowering effect of ASTX. The potential effect of ASTX on fatty acid $\beta$-oxidation is currently being investigated in our laboratory.

Hypertriacylglycerolaemia in obesity is frequently accompanied by other dyslipidaemic conditions, such as high TC and LDL-cholesterol concentrations ${ }^{(33)}$, increasing CVD risk in obese population ${ }^{(36)}$. We have previously reported that $0.03 \%$ ASTX supplementation exerts a cholesterollowering effect in $a p o E^{-/-}$mice fed a HF/high cholesterol diet $^{(13)}$. In the present study, although ASTX supplementation significantly increased the hepatic mRNA expression of LDL receptor and $H M G R$, two of the most important cholesterol regulatory genes under the transcription regulation of SREBP-2, we did not observe any changes in plasma and hepatic TC concentrations induced by ASTX supplementation. This discrepancy in findings with regard to the cholesterollowering effect of ASTX between our previous study and the present study may lie in the different animal models, i.e. $a p o E^{-/-} v$. DIO mice, as well as dietary challenges, i.e. $\mathrm{HF} /$ high cholesterol $v$. HF, used. As in the present study, DIO mice were not challenged by dietary cholesterol, a potential cholesterol-lowering effect of ASTX may not have been initiated.

Fibrosis and necroinflammation are key events in the development of NASH, and hepatic stellate cells and macrophages play a major role in the pathogenesis ${ }^{(4)}$. Upon activation by oxidative stress or fibrogenic cytokines, hepatic stellate cells transform into myofibroblasts and produce extracellular matrix proteins ${ }^{(37)}$. TGF $\beta 1$ is a cytokine that facilitates liver fibrosis ${ }^{(38)}$ and hepatocyte apoptosis ${ }^{(39)}$ and stimulates hepatic stellate cells to produce $\alpha$-smooth muscle actin and procollagen type I, $\alpha 1$, major markers of fibrosis ${ }^{(40)}$. Therefore, the effect of ASTX supplementation on the expression of fibrogenic genes was determined. Although the mRNA expression of TGF 1 was drastically elevated, that of $\alpha$-smooth muscle actin and procollagen type I, $\alpha 1$ was not significantly altered in the liver of ASTX-supplemented mice. It is known that TGF $\beta 1$ is secreted as a latent form, which requires to be cleaved by proteases such as matrix metalloproteinase- 2 and matrix metalloproteinase- 9 and to be activated by reactive oxygen species ${ }^{(41,42)}$. Therefore, the absence of $\alpha$-smooth muscle actin and procollagen type I, $\alpha 1$ induction despite an increase in TGF $\beta 1$ expression induced by ASTX supplementation indicates that the activation of TGF $\beta 1$ may be perturbed in ASTX-supplemented mice. The expression of CD68 and F4/80, macrophage markers, was not significantly altered by ASTX supplementation, suggesting that it may not have an impact on hepatic inflammation in DIO mice. However, we observed that primary splenocytes isolated from ASTXsupplemented mice exhibited a decrease in the mRNA expression of $I L-6$ upon LPS challenge compared with those isolated from control mice. Splenocytes consist of a variety 
of immune cells, including $\mathrm{T}$ and $\mathrm{B}$ lymphocytes, dendritic cells, and monocytes, and IL-6 is mainly secreted by T cells and monocytes ${ }^{(43)}$. Given that LPS stimulates primarily monocyte/macrophages, the present results indicate that ASTX supplementation may decrease the sensitivity of splenic monocytes to inflammatory stimuli.

The antioxidant effect of ASTX may play a critical role in the aforementioned beneficial effects of ASTX in DIO mice. In our previous study in $a p o E^{-/-}$mice, ASTX was found to attenuate oxidative stress in the liver by increasing the expression of $N R F-2$ and its target antioxidant genes ${ }^{(13)}$. In the present study also, we found a 2-fold increase in the mRNA expression of $N R F-2$ and an increased expression of its target endogenous antioxidant genes in the liver of ASTXsupplemented mice when compared with that in control mice. NRF-2 plays a critical role in cellular defence against oxidative stress by inducing the transcription of antioxidant genes $^{(44)}$. Under basal conditions, NRF-2 is sequestered in the cytoplasm by Kelch-like ECH-associated protein 1 (Keap1), a negative regulator of NRF-2. When cells are under oxidative stress, oxidative modifications or phosphorylations in Keap1 release NRF-2 and therefore NRF-2 enters the nucleus to activate the transcription of genes containing an antioxidant response element in their promoters ${ }^{(45)}$. Therefore, given that ASTX is known to be a potent antioxidant ${ }^{(10-12)}$, the increase in the expression of $N R F-2$ and its target genes is counterintuitive. Our observations that ASTX supplementation increased the expression of carnitine palmitoyltransferase- $1 \alpha$ and $A C O X-1$ in $a p o E^{-/-}$mice and $A C O X-1$ in DIO mice led us to speculate that ASTX may activate PPAR $\alpha$ because the genes involved in fatty acid oxidation are under the transcriptional regulation of PPAR $\alpha$ in the liver. In support of this speculation, Jia et al. ${ }^{(46)}$ demonstrated that ASTX is a PPAR $\alpha$ agonist. Therefore, the activation of PPAR $\alpha$ by ASTX could burden the mitochondrial machinery of oxidative phosphorylation due to elevated fatty acid $\beta$-oxidation, resulting in the production of reactive oxygen species. In this case, antioxidant mechanisms, possibly via the NRF-2 pathway, need to be turned on to offset the production of reactive oxygen species for the protection against oxidative damage. Further studies are required to investigate this possibility.

In conclusion, we demonstrated that $0.03 \%$ ASTX supplementation lowered plasma TAG concentrations, induced hepatic endogenous antioxidant gene expression, and attenuated inflammatory response of splenocytes in DIO mice. The effects of ASTX indicate that it may be able to prevent the development of NASH. However, as DIO mice in the present study did not develop NASH features, we were not able to determine whether ASTX supplementation could prevent obesity-associated NASH. Another mouse study is currently underway in our laboratory, in which mice are being exposed to a severe dietary challenge for a longer period of time than in the present study to induce NASH features. Nonetheless, the present study opens the possibility that ASTX could be used for preventing obesity-associated metabolic dysfunctions and inflammation.

\section{Acknowledgements}

The present study was supported by the US Department of Agriculture AFRI (2012-67018-19290; to J.-Y. L).

The authors' contributions are as follows: Y. Y. played a major role in conducting the experiments and contributed to manuscript preparation; T. X. P., C. J. W., B. K., C. S. K., and Y.-K. P. conducted the experiments; J.-Y. L. designed the experiments and contributed to the data analysis and manuscript preparation.

None of the authors has any conflicts of interest to declare.

\section{References}

1. Angulo P (2002) Nonalcoholic fatty liver disease. $N$ Engl J Med 346, 1221-1231.

2. Ludwig J, Viggiano TR, McGill DB, et al. (1980) Nonalcoholic steatohepatitis: Mayo Clinic experiences with a hitherto unnamed disease. Mayo Clin Proc 55, 434-438.

3. Rector RS, Thyfault JP, Wei Y, et al. (2008) Non-alcoholic fatty liver disease and the metabolic syndrome: an update. World J Gastroenterol 14, 185-192.

4. Marra F, Gastaldelli A, Svegliati Baroni G, et al. (2008) Molecular basis and mechanisms of progression of nonalcoholic steatohepatitis. Trends Mol Med 14, 72-81.

5. Brown WV, Fujioka K, Wilson PW, et al. (2009) Obesity: why be concerned? Am J Med 122, S4-S11.

6. Vernon G, Baranova A \& Younossi ZM (2011) Systematic review: the epidemiology and natural history of nonalcoholic fatty liver disease and non-alcoholic steatohepatitis in adults. Aliment Pharmacol Ther 34, 274-285.

7. Marchesini G, Bugianesi E, Forlani G, et al. (2003) Nonalcoholic fatty liver, steatohepatitis, and the metabolic syndrome. Hepatology 37, 917-923.

8. Hussein G, Sankawa U, Goto H, et al. (2006) Astaxanthin, a carotenoid with potential in human health and nutrition. J Nat Prod 69, 443-449.

9. Pashkow FJ, Watumull DG \& Campbell CL (2008) Astaxanthin: a novel potential treatment for oxidative stress and inflammation in cardiovascular disease. Am J Cardiol 101, 58D-68D.

10. Guerin M, Huntley ME \& Olaizola M (2003) Haematococcus astaxanthin: applications for human health and nutrition. Trends Biotechnol 21, 210-216.

11. Goto S, Kogure K, Abe K, et al. (2001) Efficient radical trapping at the surface and inside the phospholipid membrane is responsible for highly potent antiperoxidative activity of the carotenoid astaxanthin. Biochim Biophys Acta 1512, 251-258.

12. Kurashige M, Okimasu E, Inoue M, et al. (1990) Inhibition of oxidative injury of biological membranes by astaxanthin. Physiol Chem Phys Med NMR 22, 27-38.

13. Yang Y, Seo JM, Nguyen A, et al. (2011) Astaxanthin-rich extract from the green alga Haematococcus pluvialis lowers plasma lipid concentrations and enhances antioxidant defense in apolipoprotein E knockout mice. J Nutr 141, 1611-1617.

14. Lee SJ, Bai SK, Lee KS, et al. (2003) Astaxanthin inhibits nitric oxide production and inflammatory gene expression by suppressing I(kappa)B kinase-dependent NF-kappaB activation. Mol Cells 16, 97-105.

15. Uchiyama K, Naito Y, Hasegawa G, et al. (2002) Astaxanthin protects beta-cells against glucose toxicity in diabetic $\mathrm{db} / \mathrm{db}$ mice. Redox Rep 7, 290-293. 
16. Karppi J, Rissanen TH, Nyyssonen K, et al. (2007) Effects of astaxanthin supplementation on lipid peroxidation. Int $J$ Vitam Nutr Res 77, 3-11.

17. Yoshida H, Yanai H, Ito K, et al. (2010) Administration of natural astaxanthin increases serum HDL-cholesterol and adiponectin in subjects with mild hyperlipidemia. Atherosclerosis 209, 520-523.

18. Reagan-Shaw S, Nihal M \& Ahmad N (2008) Dose translation from animal to human studies revisited. FASEB J 22, 659-661.

19. Rasmussen HE, Blobaum KR, Jesch ED, et al. (2009) Hypocholesterolemic effect of Nostoc commune var. sphaeroides Kutzing, an edible blue-green alga. Eur J Nutr 48, 387-394.

20. Yang Y, Park Y, Cassada DA, et al. (2011) In vitro and in vivo safety assessment of edible blue-green algae, Nostoc commune var. sphaeroides Kützing and Spirulina platensis. Food Chem Toxicol 49, 1560-1564.

21. Rasmussen HE, Blobaum KR, Park YK, et al. (2008) Lipid extract of Nostoc commune var. sphaeroides Kutzing, a blue-green alga, inhibits the activation of sterol regulatory element binding proteins in HepG2 cells. J Nutr 138, 476-481.

22. Park YK, Rasmussen HE, Ehlers SJ, et al. (2008) Repression of proinflammatory gene expression by lipid extract of Nostoc commune var sphaeroides Kutzing, a blue-green alga, via inhibition of nuclear factor-kappaB in RAW 264.7 macrophages. Nutr Res 28, 83-91.

23. Ku CS, Pham TX, Park Y, et al. (2013) Edible blue-green algae reduce the production of pro-inflammatory cytokines by inhibiting NF- $\mathrm{BB}$ pathway in macrophages and splenocytes. Biochim Biophys Acta 1830, 2981-2988.

24. Tosello-Trampont AC, Landes SG, Nguyen V, et al. (2012) Kuppfer cells trigger nonalcoholic steatohepatitis development in diet-induced mouse model through tumor necrosis factor-alpha production. J Biol Chem 287, 40161-40172.

25. Wang C, Yu X, Cao Q, et al. (2013) Characterization of murine macrophages from bone marrow, spleen and peritoneum. BMC Immunol 14, 6.

26. Dowman JK, Tomlinson JW \& Newsome PN (2010) Pathogenesis of non-alcoholic fatty liver disease. QJM 103, 71-83.

27. Day CP \& James OFW (1998) Steatohepatitis: a tale of two "hits"? Gastroenterology 114, 842-845.

28. Tilg H \& Moschen AR (2010) Evolution of inflammation in nonalcoholic fatty liver disease: the multiple parallel hits hypothesis. Hepatology 52, 1836-1846.

29. Bellentani S, Scaglioni F, Marino M, et al. (2010) Epidemiology of non-alcoholic fatty liver disease. Dig Dis 28, 155-161.

30. Fabbrini E, Sullivan S \& Klein S (2010) Obesity and nonalcoholic fatty liver disease: biochemical, metabolic, and clinical implications. Hepatology 51, 679-689.
31. Scheig R (1996) Evaluation of tests used to screen patients with liver disorders. Prim Care 23, 551-560.

32. Malmstrom R, Packard CJ, Caslake M, et al. (1997) Defective regulation of triglyceride metabolism by insulin in the liver in NIDDM. Diabetologia 40, 454-462.

33. Subramanian S \& Chait A (2012) Hypertriglyceridemia secondary to obesity and diabetes. Biochim Biophys Acta 1821, 819-825.

34. Hassing HC, Surendran RP, Mooij HL, et al. (2012) Pathophysiology of hypertriglyceridemia. Biochim Biophys Acta 1821, 826-832.

35. Scaglioni F, Ciccia S, Marino M, et al. (2011) ASH and NASH. Dig Dis 29, 202-210.

36. Rashid S, Watanabe T, Sakaue T, et al. (2003) Mechanisms of HDL lowering in insulin resistant, hypertriglyceridemic states: the combined effect of HDL triglyceride enrichment and elevated hepatic lipase activity. Clin Biochem 36, 421-429.

37. Lee YS \& Jeong WI (2012) Retinoic acids and hepatic stellate cells in liver disease. J Gastroenterol Hepatol 27, Suppl. 2, 75-79.

38. Dooley S, Delvoux B, Lahme B, et al. (2000) Modulation of transforming growth factor beta response and signaling during transdifferentiation of rat hepatic stellate cells to myofibroblasts. Hepatology 31, 1094-1106.

39. Jang CW, Chen CH, Chen CC, et al. (2002) TGF-beta induces apoptosis through Smad-mediated expression of DAP-kinase. Nat Cell Biol 4, 51-58.

40. Bonniaud P, Margetts PJ, Ask K, et al. (2005) TGF-beta and Smad3 signaling link inflammation to chronic fibrogenesis. J Immunol 175, 5390-5395.

41. Biernacka A, Dobaczewski M \& Frangogiannis NG (2011) TGF-ßsignaling in fibrosis. Growth Factors 29, 196-202.

42. Barcellos-Hoff MH \& Dix TA (1996) Redox-mediated activation of latent transforming growth factor-beta $1 . \mathrm{Mol}$ Endocrinol 10, 1077-1083.

43. Hwang S-A, Dasgupta A \& Actor JK (2004) Cytokine production by non-adherent mouse splenocyte cultures to Echinacea extracts. Clin Chim Acta 343, 161-166.

44. Aleksunes LM \& Manautou JE (2007) Emerging role of Nrf2 in protecting against hepatic and gastrointestinal disease. Toxicol Pathol 35, 459-473.

45. Kaspar JW, Niture SK \& Jaiswal AK (2009) Nrf2:INrf2 (Keap1) signaling in oxidative stress. Free Radic Biol Med 47, 1304-1309.

46. Jia Y, Kim JY, Jun HJ, et al. (2012) The natural carotenoid astaxanthin, a PPAR-alpha agonist and PPAR-gamma antagonist, reduces hepatic lipid accumulation by rewiring the transcriptome in lipid-loaded hepatocytes. Mol Nutr Food Res 56, 878-888. 